\title{
Factors Influencing the Uptake of Canadian Research Findings into the Care of Children with Arthritis: A Healthcare Provider Perspective
}

\author{
Benjamin Rose-Davis, Janet Curran, Julia Wright, Tania Cellucci, Ciarån M. Duffy, \\ Lori B. Tucker, Michelle Batthish, Adam M. Huber, Bianca Lang, Deborah M. Levy, \\ Dax G. Rumsey, Karen N. Watanabe Duffy, and Elizabeth Stringer
}

\begin{abstract}
Objective. To determine barriers and facilitators to the uptake of findings from the Research in Arthritis in Canadian Children emphasizing Outcomes (ReACCh-Out) study into clinical care by pediatric rheumatologists (PR) and allied health professionals (AHP) caring for children with juvenile idiopathic arthritis (JIA) in Canada.

Methods. PR and AHP participated in this qualitative study through telephone interviews. Interview guides were developed using the Theoretical Domains Framework and focused on the use of information from the ReACCh-Out study in the practice of counseling patients and families. A directed content analysis approach was used for coding.

Results. Nineteen interviews (8 PR and 11 AHP) were completed. All PR had knowledge of the ReACCh-Out study. Three major themes were identified: (1) both groups are motivated to use information from research in clinical care; (2) volume and emotional effect of information on families are barriers; and (3) specific timepoints in care trigger providing this information. AHP had less knowledge of the ReACCh-Out study, did not feel it was their primary role to provide this information, and have a desire for more opportunity to participate in academic forums related to research.

Conclusion. We have described a comprehensive overview of the barriers and facilitators perceived by healthcare providers in the translation of knowledge from JIA research into use in clinical practice. These findings provide a foundation for the development of knowledge translation strategies in the care of children with JIA and other rheumatic diseases. (First Release November 15 2018; J Rheumatol 2019;46:294-300; doi:10.3899/jrheum.180282)
\end{abstract}

Key Indexing Terms:

KNOWLEDGE TRANSLATION

JUVENILE IDIOPATHIC ARTHRITIS

\section{QUALITATIVE STUDIES}

From the IWK Health Centre and Dalhousie University, Halifax, Nova Scotia; Children's Hospital of Eastern Ontario and University of Ottawa, Ottawa, Ontario; British Columbia Children's Hospital, Division of Rheumatology, and University of British Columbia, Vancouver, British Columbia; McMaster Children's Hospital, Division of Rheumatology, and McMaster University, Hamilton; Hospital for Sick Children and University of Toronto, Toronto, Ontario; Stollery Children's Hospital and University of Alberta, Edmonton, Alberta, Canada.

Supported by a Canadian Initiative for Outcomes in Rheumatology cAre (CIORA) grant.

B. Rose-Davis, BA, IWK Health Centre; J. Curran, BN, MEd, PhD, Clinician Scientist, Pediatrics and Emergency Medicine, Associate Professor of Nursing, IWK Health Centre; J. Wright, BSc, Dalhousie University; T. Cellucci, MD, FRCPC, MSCCH, Pediatric Rheumatologist, Associate Professor of Pediatrics, McMaster University; C.M. Duffy, MB, BCh, MSc, FRCPC, FRCPI, Pediatric Rheumatologist, Professor of Pediatrics, Children's Hospital of Eastern Ontario; L.B. Tucker, MD, FRCPC, Pediatric Rheumatologist, Professor of Pediatrics, British Columbia Children's Hospital, Division of Rheumatology; M. Batthish, MSc, MD, FRCPC, Pediatric Rheumatologist, Assistant Professor of Pediatrics, McMaster Children's Hospital, Division of Rheumatology; A.M. Huber, MD, MSc, FRCPC, Pediatric Rheumatologist, Professor of Pediatrics, IWK Health Centre; B. Lang, MD, FRCPC, Pediatric Rheumatologist, Professor of Pediatrics, IWK Health Centre; D.M. Levy, MD, MS, FRCPC, Pediatric Rheumatologist, Associate Professor of Pediatrics, Hospital for Sick Children and University of Toronto;
D.G. Rumsey, MD, FRCPC, Pediatric Rheumatologist, Assistant Professor of Pediatrics, University of Alberta; K.N. Watanabe Duffy, MD, FRCPC, Pediatric Rheumatologist, Associate Professor of Pediatrics, Children's Hospital of Eastern Ontario; E. Stringer, MD, MSc, FRCPC, Pediatric Rheumatologist, Associate Professor of Pediatrics, IWK Health Centre. Address correspondence to Dr. E. Stringer, Division of Rheumatology, IWK Health Centre, 5850 University Ave., Halifax, Nova Scotia B3K 6R8, Canada.E-mail: Elizabeth.stringer@iwk.nshealth.ca

Accepted for publication September 6, 2018.

Juvenile idiopathic arthritis (JIA) is the most common chronic rheumatic disease of childhood, with an estimated prevalence of $1-4 / 1000$ children ${ }^{1}$. Every child with JIA is at risk of experiencing disability, pain, decreased quality of life, and prolonged requirements for medications ${ }^{2,3,4}$. Adolescents are also greatly affected and describe feelings of isolation, physical and emotional pain, and uncertainty about the future ${ }^{5}$. Throughout the disease course, parents experience an "emotional roller coaster" associated with the unpredictable nature of JIA and worry about medication side effects $^{6,7,8}$. The experience of patients and caregivers over the disease trajectory of JIA can be very challenging. 
Research teams studying outcomes in JIA have assembled cohorts of patients around the world, including the ReACCh-Out study (Research on Arthritis in Canadian Children, emphasizing Outcomes ${ }^{2,9}$. A primary aim of the ReACCh-Out study was to summarize current practice knowledge for healthcare providers to use when counseling children and families about outcomes early in the disease course. We hypothesize that having this information early in the disease course could help to inform decision making and help to reduce some of the distress associated with worrying about an uncertain future. The uptake of this information by healthcare providers for use in counseling in clinical care is unknown.

Having research findings used by healthcare providers, patients, or caregivers when making important health-related decisions is a critical outcome in patient-oriented research ${ }^{10}$. This is also known as knowledge translation (KT); many KT models suggest that KT should take place through an iterative, dynamic process involving key groups ${ }^{10}$. A crucial part of this process is to identify existing barriers and facilitators that specifically affect the translation of knowledge from a research study into clinical care or other relevant contexts.

The objective of our study was to determine the barriers and facilitators to the uptake of findings from the ReACCh-Out study that are perceived by 2 groups: (1) pediatric rheumatologists (PR); and (2) allied health professionals (AHP). We will combine this knowledge with findings from a parallel study of adolescents and parents, to design future KT interventions ${ }^{11}$.

\section{MATERIALS AND METHODS}

We conducted individual telephone interviews with PR and AHP caring for children with JIA from across Canada. Potential participants were recruited by an e-mail sent to them by their respective professional groups or PR division heads.

Demographic characteristics including years in practice, region, and whether they practiced in a community or academic setting were collected for use in purposeful sampling. We aimed to enroll 6-10 participants per group based on previous estimates for achieving thematic saturation in qualitative studies ${ }^{12,13}$. Ethics approval for our study was granted by the Research Ethics Board of the IWK Health Centre (1020567).

All interviews were conducted between October and December 2016 by a trained research coordinator using a semistructured interview guide. Verbal consent was obtained for each participant prior to beginning the interview. Interview guides were developed using the Theoretical Domains Framework (TDF) ${ }^{14}$ and focused on the behavior of interest: healthcare providers' use of information from the ReACCh-Out study in the counseling of patients and families. The TDF is a validated framework that integrates 33 behavior change theories into 14 theoretical domains. The core interview questions were the same for all participants but were tailored according to the participant's level of knowledge of Canadian JIA research. Interview questions for participants with knowledge of the ReACCh-Out study centered on those results. Questions for others with less knowledge focused on the wider body of JIA literature, and questions for those with little knowledge of JIA research focused on research in general. The domains of the TDF with sample questions from the interviews are shown in Table 1.

Two independent coders (BRD, ES) completed the analysis using NVivo11 software (QSR International Pty Ltd. Version 11, 2015). We used a directed content analysis approach to code relevant statements from the transcripts into the domains of the $\mathrm{TDF}^{15}$. Each reviewer independently coded each transcript using the TDF as a coding framework. To ensure consistency in coding, the reviewers met after independently coding the first and second transcripts from the PR and AHP interviews, respectively. The reviewers then met after coding 2-3 additional transcripts from the respective groups. At each meeting, the reviewers shared their coding results and resolved any differences through consensus. Recruitment and analysis continued until no new additional data were found that developed aspects of a conceptual category (e.g., a TDF domain). Following the directed content analysis, inductive coding was then performed independently by each reviewer to create a list of specific beliefs within each domain, which were then integrated through discussion. A specific belief was defined as a collection of responses from more than 1 interview participant with an underlying theme that influenced the behavior of interest. Finally, relevant themes were identified based on whether they were mentioned across at least $50 \%$ of participants and their likelihood of affecting the use of research in practice and/or the ease with which they could be targeted (e.g., overcome barriers, leverage facilitators) in KT interventions.

\section{RESULTS}

Nineteen PR and 16 AHP expressed interest in participating in the study and provided their demographic information. Eight PR and 11 AHP were selected for interviews; the selection was based on maximizing the variability in the characteristics presented in Table 2. The interviews lasted an average of $25.5 \mathrm{~min}$ (range 17.4-48.4). All PR had knowledge of the ReACCh-Out study. Of the AHP, 4/11 reported knowledge of the results of the ReACCh-Out study, 4/11 knew only that the study existed, and 3/11 were not aware of the study.

We identified 4 themes common to the PR and AHP groups: motivation to use information from research in clinical care; barriers related to volume of information and lack of time; the barrier of the emotional effect of information; and that specific timepoints in care are triggers for sharing research-related information. Barriers exclusive to the AHP group formed our final theme. The 5 themes are described below with the corresponding TDF domains for each theme and illustrative quotes. The quotes are denoted as attributable to a "PR" or an "AHP" and a corresponding participant number.

Motivation to use information from research in clinical care (relevant TDF domains: beliefs about consequences, optimism, intentions). Motivation is a key component of behavior change as described by the Behaviour Change Wheel, a framework used to guide the development and evaluation of behavior change interventions ${ }^{16}$. The TDF domains represented by PR and AHP within this theme (beliefs about consequences, optimism, intentions) have been mapped onto the motivation component of the Behaviour Change Wheel because they contribute positively toward motivation. For example, PR and AHP believed that providing information from research to patients and families could result in positive health outcomes including reduced fear, improved coping, improved adherence to treatments, better decision making, and overall better medical care.

Personal non-commercial use only. The Journal of Rheumatology Copyright @ 2019 . All rights reserved. 
Table 1. TDF domains and semistructured interview questions.

Domain Name (definition)

Knowledge (An awareness of the existence of something)

Intentions (A conscious decision to perform a behavior or a resolve to act in a certain way)

Skills (An ability or proficiency acquired through practice)

Goals (Mental representations of outcomes or end states that an individual wants to achieve)

Social/professional role and identity (A coherent set of behaviors and displayed personal qualities of an individual in a social or work setting)

Memory, attention, and decision process (The ability to retain information, focus selectively on aspects of the environment, and choose between 2 or more alternatives)

Beliefs about capabilities (Acceptance of the truth, reality, or validity about an ability, talent, or facility that a person can put to constructive use)

Environment, context, and resources (Any circumstance of a person's situation or environment that discourages or encourages the development of skills and abilities, independence, social competence, and adaptive behavior)

Beliefs about consequences (Acceptance of the truth, reality, or validity about outcomes of a behavior in a given situation)

Social influences (Those interpersonal processes that can cause individuals to change their thoughts, feelings, or behaviors)

Optimism (The confidence that things will happen for the best or that desired goals will be attained)

Emotion (A complex reaction pattern, involving experiential, behavioral, and physiological elements, by which the individual attempts to deal with a personally significant matter or event)

Reinforcement (Increasing the probability of a response by arranging a dependent relationship, or contingency, between the response and a given stimulus)

Behaviour regulation (Anything aimed at managing or changing objectively observed or measured actions)
Sample Question from Interview

Are you familiar with any Canadian research on outcomes in JIA?

Do you usually intend to provide information about the future to patients and families?

What skills are needed to use this information?

How important is it to provide the most up-to-date information on the outcomes of JIA to patients and parents?

Do you feel it is your role to have discussion with patients and families about recent research findings?

What triggers your decision to use information from the ReACCh-Out study?

How confident do you feel in your ability to use information from the ReACCh-Out study in counseling patients and families about future disease course?

What factors in your clinical environment influence your decision to use information from the ReACCh-Out study?

What do you think is the balance of potential benefits and potential harms for providing information about the future to patients and parents?

Do other healthcare providers in your division influence your decision to use information from Canadian JIA research?

Do you feel that the provision of information obtained from the ReACCh-Out study will improve patient care?

Does providing information to patients and caregivers ever evoke an emotional response in you?

Are there any incentives to providing information about the future to patients and families?

What do you think is needed to ensure that you consistently use information obtained from the ReACCh-Out study or other research on JIA in the clinic setting?

TDF: Theoretical Domains Framework; JIA: juvenile idiopathic arthritis; ReACCh-Out: Research in Arthritis in Canadian Children emphasizing Outcomes study.

PR (1): It just helps them prepare for and anticipate the future ... it could potentially keep them motivated to be adherent to the medications.

AHP (6): I think knowledge is $100 \%$ power for everybody and their fears are less because they know what they can expect.

Second, the PR and AHP with knowledge of the ReACCh-Out study indicated they intend to incorporate the research findings into the clinical care of patients and families.
PR (5): I try to [incorporate research findings from ReACCh-Out] to the best of my ability, let them know what's going to happen down the road: is it a chronic condition, is it not, is it relapsing, is it not? ... I try to give them some idea of what it looks like for them.

AHP (4): The way that we are best able to give information ... is based on looking at a large number of kids and telling you [the parent] on average what happens ... this is the best information we can give ... the most current data ... 
Table 2. PR and AHP respondent characteristics.

\begin{tabular}{lccc}
\hline & Participants & PR, $\mathrm{n}=8$ & AHP, $\mathrm{n}=11$ \\
\hline \multirow{2}{*}{ Role } & PT & - & 6 \\
& RN & - & 3 \\
\multirow{2}{*}{ Experience, yrs } & OT & - & 2 \\
& $<5$ & 3 & 3 \\
& $6-10$ & 1 & 2 \\
FTE, \% & $11-20$ & 2 & 2 \\
& $>20$ & 2 & 3 \\
& $<25$ & - & 3 \\
Center* & $>25-50$ & - & 3 \\
& $>50-75$ & - & 1 \\
Region** & $>75$ & - & 3 \\
& Community & 0 & 2 \\
& Academic & 8 & 9 \\
& Eastern & 1 & 4 \\
& Central & 4 & 6 \\
& Western & 3 & 1
\end{tabular}

* Overall, 9 academic pediatric rheumatology centers are represented; 2 are community-based. ** Regions are defined as: Eastern Canada (Newfoundland, Nova Scotia, New Brunswick, Prince Edward Island), Central Canada (Quebec and Ontario), and Western Canada (Manitoba, Saskatchewan, Alberta, and British Columbia); across these regions 6 provinces were represented. PR: pediatric rheumatologist; AHP: allied health professional; PT: physiotherapist; RN: registered nurse; OT: occupational therapist; FTE: full-time equivalent.

and let them know that we were part of the ReACCh-Out study.

Barriers related to volume of information and lack of time (relevant TDF domains: memory, attention and decision processes, environmental context, resources). Three interrelated items emerged as barriers to incorporating information from research into clinical care from the perspectives of both PR and AHP: difficulty with recall, lack of time, and shortage of memory aids. In relation to the ReACCh-Out study, the majority of PR found it difficult to recall specific results from the study, despite more than half identifying as ReACCh-Out study investigators. AHP also cited memory as a general barrier for them although the majority also did not have any specific knowledge about the ReACCh-Out study results.

PR (5): The manuscript itself is very information-heavy ... I think that reading it is all fine and well but to keep the numbers at hand is hard.

AHP (7): Right off the top of my head I might not remember all the specifics of the research.

Lack of time had 2 meanings for PR and AHP. Both groups cited limited time to search for and read new publications in the literature as a barrier. The time available to spend with patients and families in the clinic was also felt to be a barrier to incorporating research findings into the clinical encounter.

PR (2): Taking the time to synthesize the data and then to be able to create the necessary dialogue with families. I think that is probably more of the barrier for me.
AHP (2): We don't always have time to go and look for the most current information.

PR (7): If I'm behind in my schedule, running late then I'd be less likely to talk about the research.

Half of the PR and 2/11 AHP cited a lack of tools to complement and/or facilitate verbal discussions (e.g., pamphlets, visuals, Websites, etc.) when providing information from research to patients and families. We categorized that as a resource-related barrier.

PR (1): Sometimes I guess if the information is very technical it is a bit hard to put into layman's terms, so maybe some aid, like visual aids to help with that.

PR (7): It would be very helpful to me to have like some kind of knowledge translation tool to be able to practice it better, I think.

AHP (9): If there was a cheat sheet ... [it] would be easier to put that into practice and then it's a reminder for me.

The barrier of perceived emotional effect of information (relevant TDF domains: environmental context and resources). PR and AHP had the perception that families were sometimes too overwhelmed or upset to process large amounts of information and this could modify their approach to counseling.

PR (2): They are not in the right frame of mind to have that kind of discussion. They are too upset or overwhelmed.

AHP (6): For some, too much information is overwhelming.

Specific timepoints in care are triggers for sharing researchrelated information (relevant TDF domains: environmental context and resources, behavioral regulation). Timepoints during the illness trajectory (new diagnosis, discussing new treatments, and counseling about the future) are all specific times during which PR and AHP were most likely to include information from relevant research, including the ReACChOut study.

PR (4): That happens in 3 different situations. One is when I am making a new diagnosis of a patient and trying to tell the families what is going to happen. Second is when I'm prescribing treatments for patients with arthritis or other conditions and trying to describe the possible things that are going to happen. Third is when families ask me about what the future holds and why we make certain decisions.

AHP (4): Any sort of change in clinical status like a change in therapy, then for sure, that's probably the most common time that I would be accessing research.

AHP-only barriers (relevant TDF domains: social/professional role and identity, knowledge, and social influences). Four out of 11 AHP had knowledge of specific findings of the ReACCh-Out study, while the remainder only knew of its existence or had no knowledge of it. Lack of being up-to-date on the latest information from research was demonstrated.

AHP (1): In the last while I have not been up on the research.

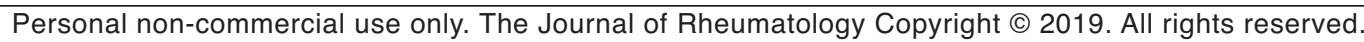


When asked about their perceived role in communicating specific results about outcomes from the ReACCh-Out study, AHP said they did not feel that was one of their primary roles in the care of children with JIA.

AHP (5): I think actually discussing those outcomes ... would be more owned by the rheumatologist.

AHP looked to the other members on the care team, particularly the PR, for updates around research in JIA. They had a desire for more avenues and time spent on sharing information from research, particularly how the research could affect clinical care.

AHP (7): I would say, you know when we do our academic rounds, you know or rounds at the hospital our docs do bring forth that. Probably I would say more so from our docs is where I get most of that information on research.

\section{DISCUSSION}

This is the first study, to our knowledge, to determine barriers and facilitators to the translation of knowledge gained from research into the clinical care of children with JIA from the perspectives of healthcare providers. We studied this in a Canadian context, using findings from a national collaborative research project: the ReACCh-Out study. We interviewed pediatric rheumatologists, physical and occupational therapists, and nurses caring for children with JIA from across Canada.

In healthcare research, it is well established that the translation of findings from clinical studies into practice often fails, resulting in the patients and families not benefiting from newly discovered information that could improve care ${ }^{17}$. Many factors can influence the uptake of research information into clinical care. We used the TDF as the foundation for the development and analysis of our study to examine barriers and enablers of knowledge uptake in this context ${ }^{14}$. The TDF has been used across multiple disciplines to help understand behaviors of healthcare providers, as well as patients ${ }^{18,19}$

Our findings show that PR and AHP believe that providing information from research to patients and families can result in positive health outcomes, and most intend to incorporate the provision of information from research into clinical care. Enthusiasm for the incorporation of evidence from research into clinical care is something that could be leveraged in relation to future involvement in designing, implementing, and evaluating KT interventions within the Canadian pediatric rheumatology community.

Most PR found it difficult to recall specific results from the ReACCh-Out study, despite more than half identifying as ReACCh-Out investigators; AHP cited memory as a general barrier for them. PR/AHP said they felt that a KT intervention or tool (e.g., a plain-language summary or "cheat sheet") could overcome this barrier, and having this available could act as a reminder to use this information in practice. In a recent study of incorporating physical activity guidelines into practice, a lack of pragmatic tools in the environment was also a barrier for physicians and nurse practitioners ${ }^{20}$. There is evidence supporting the incorporation of reminders into clinical care as a strategy to change physician behavior and processes of care ${ }^{21}$. Future work, therefore, could be directed at designing KT tools that serve to both increase knowledge and act as a reminder to healthcare providers. For example, research findings could be summarized and disseminated through a Web-based portal with automatic notifications sent to users depending on user preference. The McMaster Optimal Aging Portal is an example of this approach and is aimed at clinicians, policy makers, and the public ${ }^{22,23}$.

Lack of time as a barrier to incorporating evidence-based care into practice has been cited by a range of health professionals across different clinical contexts ${ }^{20,24,25,26}$. Boerner, et al found that lack of time within sessions was the second most commonly reported barrier to providing evidence-based sleep-related care to families by nurses, physicians, psychologists, and social workers ${ }^{24}$. In a recent study of rehabilitation clinicians and occupational and physical therapists, lack of time was a major barrier to searching and interpreting the relevant literature. The themes around lack of time that emerged from our study mirror these previous findings ${ }^{27}$. Interventions addressing efficient ways of keeping abreast of relevant research results could be beneficial, especially for PR/AHP who spend only part of their time caring for children with JIA and/or work outside an academic setting. Another priority for future research should be to understand how best to incorporate information from research both within and outside the constraints of the clinic visit.

The final barrier cited by both PR and AHP was the perception that families are sometimes too overwhelmed or upset to process large amounts of information. Indeed, we have found that parents are quite overwhelmed by information presented to them by the healthcare team at certain times during their child's illness, particularly at diagnosis and during flares ${ }^{11}$. Asking patients about information preferences was suggested by Bernacki, et al in a review of best practices for communication about serious illness goals (e.g., "How much information about what is likely to be ahead with your illness would you like from me?") ${ }^{28}$. Rather than assuming that families are too overwhelmed, seeking preferences from patients and families around their goals and desire for information, including information from research, could enhance the delivery of information.

The clearest triggers for PR for sharing information from research, including the ReACCh-Out study, were specific timepoints during the illness trajectory: new diagnosis, discussing new treatments, and counseling about the future. This corresponds with what we have found about parents' information needs when caring for their children ${ }^{11}$. Therefore, a possible facilitator would be to focus KT interventions at specific times that are relevant for both the PR/AHP and parent. It will be critical to consider how this

Personal non-commercial use only. The Journal of Rheumatology Copyright @ 2019. All rights reserved. 
information is delivered, however, because conventional verbal dissemination in the clinic may be overwhelming. This ties in with the description commonly cited in KT spheres: "knowledge translation is about getting the right information, to the right people, at the right time, and in a format they can use, so as to influence decision making ${ }^{29}$." Patient decision aids (PtDA) have been developed to facilitate decision making and help to address this need. PtDA, which can be delivered through a range of approaches (paper-based, Web-based), help by making explicit the decision that needs to be made, providing information about the options and outcomes, and by clarifying personal values. Use of PtDA can result in patients feeling more knowledgeable, better informed, and clearer about their values ${ }^{30}$. PtDA have been studied in the care of adults with rheumatic disease such as rheumatoid arthritis $31,32,33,34$. Within the Pediatric Rheumatology Care and Outcomes Improvement Network, Brinkman, et al developed medication choice cards among patients, parents, and PR/AHP ${ }^{35}$. While user acceptability of the decision aid was high, reliable implementation into clinical care was challenging. Future research should be directed at understanding the barriers to implementing interventions such as decision aids and developing innovative modalities for information delivery and use.

It is also important to consider including all relevant persons early in the research process. This is highlighted by the finding that the majority of AHP had little to no knowledge about the results of the ReACCh-Out study. Although AHP indicated that they did not necessarily feel it was their primary role to communicate this information to patients and families, this information is likely highly relevant to them.

It is important to consider the potential limitations of our study. It is possible that the participants interviewed were more interested in research and/or KT than nonresponders, resulting in a selection bias. Indeed, the majority of PR responders were ReACCh-Out investigators. We speculate that this bias could have led to barriers being underestimated or missed. Most PR and AHP in the study worked in academic centers as opposed to community practices. This is likely reflective of the model of care for pediatric rheumatology in Canada, where most children with JIA receive most or part of their care in university-based clinics. We also anchored our interviews around the ReACCh-Out study, which could affect generalizability. However, despite these limitations, the general themes identified in our work form a valuable foundation for future initiatives in KT research and KT planning.

Our study demonstrates that while healthcare providers are motivated to incorporate information from research into clinical care, significant barriers exist. These included a lack of time (both to search the literature for new research and to share research results in the clinic), the challenge of distilling and recalling large volumes of information, a paucity of memory aids and KT tools for use during clinical encounters, and concern regarding overburdening already overwhelmed patients and parents with information. This information will be used, together with input from patients and caregivers, to design KT interventions and implementation strategies for use in the care of children with JIA.

\section{REFERENCES}

1. Gowdie PJ, Tse SM. Juvenile idiopathic arthritis. Pediatr Clin North Am 2012;59:301-27.

2. Guzman J, Oen K, Tucker LB, Huber AM, Shiff N, Boire G, et al. The outcomes of juvenile idiopathic arthritis in children managed with contemporary treatments: results from the ReACCh-Out cohort. Ann Rheum Dis 2015;74:1854-60.

3. Oen K, Guzman J, Dufault B, Tucker LB, Shiff NJ, Watanabe Duffy $\mathrm{K}$, et al. Health-related quality of life in an inception cohort of children with juvenile idiopathic arthritis: a longitudinal analysis. Arthritis Care Res 2018;70:134-44.

4. Rumsey DG, Guzman J, Rosenberg AM, Huber AM, Scuccimarri R, Shiff NJ, et al. Characteristics and course of enthesitis in a juvenile idiopathic arthritis inception cohort. Arthritis Care Res 2018;70:303-8.

5. Stinson JN, Feldman BM, Duffy CM, Huber AM, Tucker LB, McGrath PJ, et al. Jointly managing arthritis: Information needs of children with juvenile idiopathic arthritis (JIA) and their parents. J Child Health Care 2012;16:124-40.

6. Gomez-Ramirez O, Gibbon M, Berard R, Jurencak R, Green J, Tucker L, et al. A recurring rollercoaster ride: A qualitative study of the emotional experiences of parents of children with juvenile idiopathic arthritis. Pediatr Rheumatol Online J 2016;14:13.

7. Lipstein EA, Lovell DJ, Denson LA, Kim SC, Spencer C, Ittenbach RF, et al. High levels of decisional conflict and decision regret when making decisions about biologics. J Pediatr Gastroenterol Nutr 2016;63:e176-e81.

8. Guzman J, Oen K, Huber AM, Watanabe Duffy K, Boire G, Shiff N, et al. The risk and nature of flares in juvenile idiopathic arthritis: results from the ReACCh-Out cohort. Ann Rheum Dis 2016;75:1092-8.

9. Beukelman T, Anink J, Berntson L, Duffy C, Ellis JA, Glerup M, et al. A survey of national and multi-national registries and cohort studies in juvenile idiopathic arthritis: Challenges and opportunities. Pediatr Rheumatol Online J 2017;15:31.

10. Curran JA, Grimshaw JM, Hayden JA, Campbell B. Knowledge translation research: The science of moving research into policy and practice. J Contin Educ Health Prof 2011;31:174-80.

11. Wright J, Rose-Davis B, Curran J, Cellucci T, Duffy C, Tucker L, et al. Knowledge translation in juvenile idiopathic arthritis in Canada: a focus on parents of children with JIA [abstract]. Arthritis Rheumatol 2017;69:abstract 2317.

12. Malterud K, Siersma VD, Guassora AD. Sample size in qualitative interview studies: guided by information power. Qual Health Res 2015 Nov 27 (E-pub ahead of print).

13. Francis JJ, Johnston M, Robertson C, Glidewell L, Entwistle V, Eccles MP, et al. What is an adequate sample size? Operationalising data saturation for theory-based interview studies. Psychol Health 2010;25:1229-45.

14. Cane J, O'Connor D, Michie S. Validation of the theoretical domains framework for use in behaviour change and implementation research. Implement Sci 2012;7:37.

15. Hsieh HF, Shannon SE. Three approaches to qualitative content analysis. Qual Health Res 2005;15:1277-88.

16. Michie S, Atkins L, West R. The behaviour change wheel: a guide to designing interventions. London: Silverback Publishing; 2014.

Personal non-commercial use only. The Journal of Rheumatology Copyright @ 2019 . All rights reserved. 
17. Grimshaw JM, Eccles MP, Lavis JN, Hill SJ, Squires JE. Knowledge translation of research findings. Implement Sci 2012;7:50.

18. Phillips CJ, Marshall AP, Chaves NJ, Jankelowitz SK, Lin IB, Loy $\mathrm{CT}$, et al. Experiences of using the theoretical domains framework across diverse clinical environments: a qualitative study. J Multidiscip Healthc 2015;8:139-46.

19. Atkins L, Francis J, Islam R, O'Connor D, Patey A, Ivers N, et al. A guide to using the theoretical domains framework of behaviour change to investigate implementation problems. Implement Sci 2017;12:77.

20. Clark RE, McArthur C, Papaioannou A, Cheung AM, Laprade J, Lee L, et al. "I do not have time. Is there a handout I can use?": Combining physicians' needs and behavior change theory to put physical activity evidence into practice. Osteoporos Int 2017;28:1953-63.

21. Cheung A, Weir M, Mayhew A, Kozloff N, Brown K, Grimshaw J. Overview of systematic reviews of the effectiveness of reminders in improving healthcare professional behavior. Syst Rev 2012;1:36.

22. Barbara AM, Dobbins M, Brian Haynes R, Iorio A, Lavis JN, Raina $\mathrm{P}$, et al. McMaster Optimal Aging Portal: an evidence-based database for geriatrics-focused health professionals. BMC Res Notes 2017;10:271.

23. Neil-Sztramko SE, Farran R, Watson S, Levinson AJ, Lavis JN, Iorio A, et al. If you build it, who will come? A description of user characteristics and experiences with the McMaster Optimal Aging Portal. Gerontol Geriatr Med 2017;3:1-9.

24. Boerner KE, Coulombe JA, Corkum P. Barriers and facilitators of evidence-based practice in pediatric behavioral sleep care: qualitative analysis of the perspectives of health professionals. Behav Sleep Med 2015;13:36-51.

25. Heiwe S, Kajermo KN, Tyni-Lenne R, Guidetti S, Samuelsson M, Andersson IL, et al. Evidence-based practice: attitudes, knowledge and behaviour among allied health care professionals. Int J Qual Health Care 2011;23:198-209.

26. Jette DU, Bacon K, Batty C, Carlson M, Ferland A, Hemingway $\mathrm{RD}$, et al. Evidence-based practice: beliefs, attitudes, knowledge, and behaviors of physical therapists. Phys Ther 2003;83:786-805.
27. Patel D, Koehmstedt C, Jones R, Coffey NT, Cai X, Garfinkel S, et al. A qualitative study examining methods of accessing and identifying research relevant to clinical practice among rehabilitation clinicians. J Multidiscip Healthc 2017;10:429-35.

28. Bernacki RE, Block SD, American College of Physicians High Value Care Task Force. Communication about serious illness care goals: a review and synthesis of best practices. JAMA Intern Med 2014;174:1994-2003.

29. What is knowledge translation? [Internet. Accessed October 16, 2018.] Available from: www.ktaustralia.com/\#What

30. Madden K, Kleinlugtenbelt YV. Cochrane in corr ((r)): Decision aids for people facing health treatment or screening decisions. Clin Orthop Relat Res 2017;475:1298-304.

31. Barton JL, Trupin L, Schillinger D, Evans-Young G, Imboden J, Montori VM, et al. Use of low-literacy decision aid to enhance knowledge and reduce decisional conflict among a diverse population of adults with rheumatoid arthritis: results of a pilot study. Arthritis Care Res 2016;68:889-98.

32. Li LC, Adam PM, Backman CL, Lineker S, Jones CA, Lacaille D, et al. Proof-of-concept study of a Web-based methotrexate decision aid for patients with rheumatoid arthritis. Arthritis Care Res 2014;66:1472-81.

33. Li LC, Shaw CD, Lacaille D, Yacyshyn E, Jones CA, Koehn C, et al. Effects of a Web-based patient decision aid on biologic and small-molecule agents for rheumatoid arthritis: results from a proof-of-concept study. Arthritis Care Res 2018;70:343-52.

34. Martin RW, Brower ME, Geralds A, Gallagher PJ, Tellinghuisen DJ . An experimental evaluation of patient decision aid design to communicate the effects of medications on the rate of progression of structural joint damage in rheumatoid arthritis. Patient Educ Couns 2012;86:329-34.

35. Brinkman WB, Lipstein EA, Taylor J, Schoettker PJ, Naylor K, Jones K, et al. Design and implementation of a decision aid for juvenile idiopathic arthritis medication choices. Pediatr Rheumatol Online J 2017;15:48 